\title{
Three-Dimensional Metallography for Visualization, Characterization, Modeling, and Simulation of Three-Dimensional Microstructures
}

\author{
A. M. Gokhale, H. Singh, Y. Mao, S. Zhang, and A. Gurumurthy \\ School of Materials Science and Engineering, Georgia Institute of Technology \\ Atlanta, Georgia-30332
}

It is the central principle of materials science that processing governs microstructure and the microstructure influences the properties and performance of materials. Consequently, quantitative characterization and mathematical representation of microstructure are of considerable importance in materials science. Material microstructures are three-dimensional and, therefore, the attributes of three-dimensional microstructural geometry are of core interest. Nonetheless, as most of the materials are opaque, the microstructural observations are usually on the two-dimensional (2D) metallographic sections through the three-dimensional (3D) microstructural domains of interest. The microstructure observed in a metallographic section consists of intersections of the features in the 3D microstructure with the sectioning plane. Therefore, in a metallographic plane, the volumes (e.g., grains, voids, particles) in a 3D microstructure appear as areas, and the surfaces (e.g., grain boundaries, precipitate interfaces) appear as lines. Clearly, a 2D metallographic section does not contain all the information concerning the true 3D microstructural geometry. Consequently, development and applications of the techniques for reconstruction, visualization, and direct quantitative characterization of opaque 3D microstructures (i.e., 3D metallography) are of considerable interest.

It is well known that material microstructures are of stochastic nature. Consequently, no two microstructures are exactly alike; no two fields of view in a microstructure are exactly alike; and in most microstructures, no two particles/grains in a field of view are exactly alike. It follows that microstructure characterization must be couched in statistical terms. Further, microstructural features have complex morphologies, their locations and orientations are often non-uniform, and spatial clustering and correlations frequently exist. Therefore, to be useful, a reconstructed 3D microstructure volume segment must have high resolution (to clearly resolve intricate complex 3D morphologies of the features) and large volume (to be regarded as a statistical representative volume segment), which are conflicting requirements. This is because in traditional microscopy, the size of the microstrucutral field of view is inversely proportional to square of the magnification: higher the resolution/magnification smaller is the field of view. This difficulty can be resolved by using the montage serial sectioning technique developed at Georgia Tech that combines modern digital image processing, serial sectioning, and microscopy. In this invited lecture, applications of montage serial sectioning will be presented in the context of reconstruction, visualization, and quantitative characterization of high-resolution large-volume segments of 3D microstructures of metal matrix composites, cast Mg-alloys, and cast Al-alloys. The techniques for quantitative characterization of these reconstructed 3D microstructures using digital image analysis and stereological techniques are also presented. The reconstructed 3D microstructures have been used to create digital libraries of large number of individual microstructural features (particles, pores, inclusions, etc) present in the 3D microstructures. Utility of these digital libraries for computer simulations of realistic 3D microstructures and rational virtual $3 \mathrm{~d}$ microstructures are discussed. Figures 1 and 2 illustrate application of the 
montage serial sectioning technique for reconstruction of 3D microstructure of multi-phase multi-scale cast Al-Si base alloy.

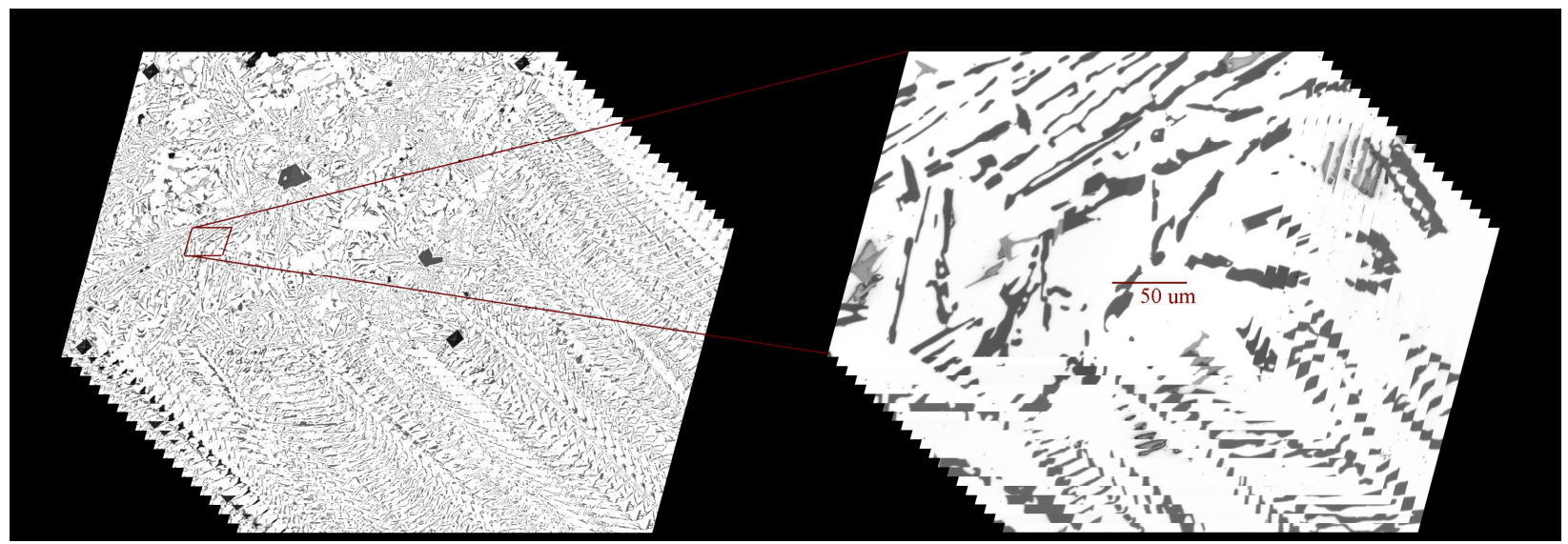

Figure 1: Left: Stack of aligned 20 montage serial sections of microstructure. Each serial section montage contains 49 contiguous microstructural fields. Right: The magnified view of the small bordered region of the stack of 20 montage serial sections of the microstructure in the left.

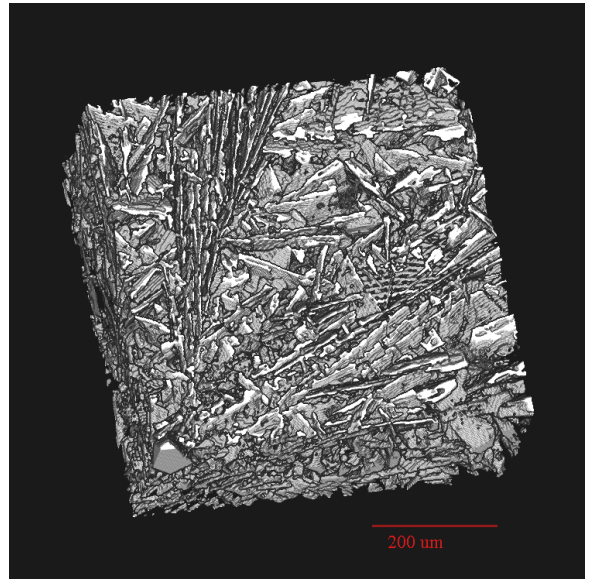

(a)

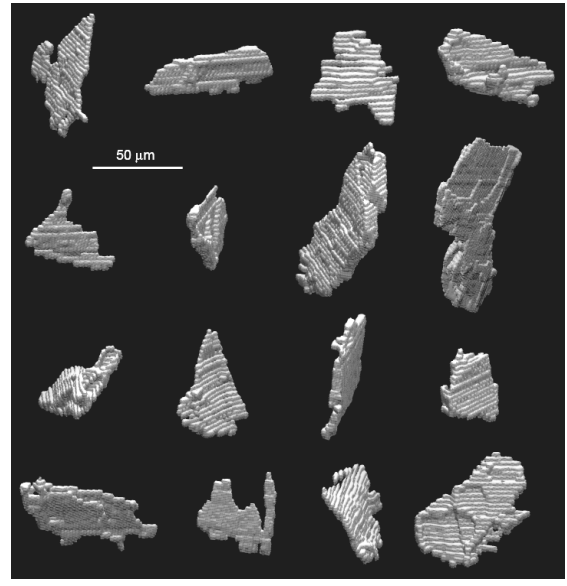

(b)

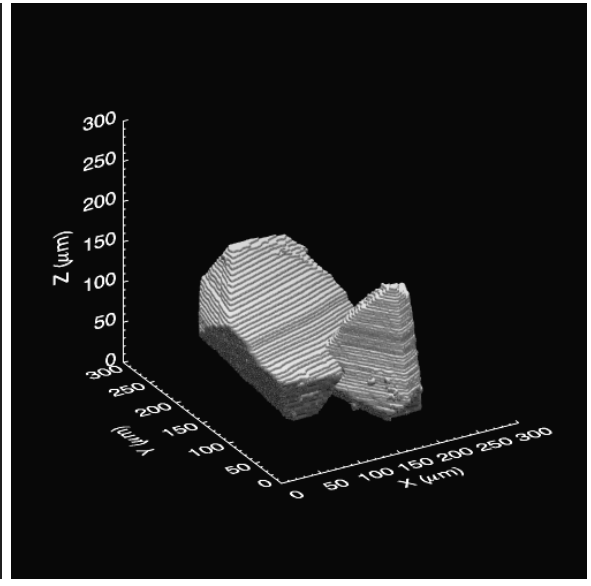

(c)

Figure 2(a) Surface-rendered 3D microstructure segment depicting eutectic Si platelets. (b) A part of the digital library of individual eutectic Si platelets extracted from 3D microstructure. (c) A primary Si idiomornh extracted from the $3 \mathrm{D}$ microstructure 